\title{
Perceptions of Saudi Arabian adults towards food and health: careless or clueless?
}

\author{
M.M. Alkhalaf, C.A. Edwards and E. Combet \\ Human nutrition new lister building, School of Medicine, College of Medical, Veterinary and Life Sciences, University \\ of Glasgow, Glasgow, G31 2ER, UK
}

Rising incidence of non-communicable diseases (NCDs) in Saudi Arabia (SA), including cardiovascular diseases (CVDs) and Type 2 Diabetes Mellitus (T2DM), has coincided with dramatic changes in socioeconomic status and lifestyle factors, including unhealthy dietary habits ${ }^{(1)}$. However, many of the associated risk factors are modifiable ${ }^{(2)}$. An improved diet, increased physical activity and weight loss can be comparable, or sometime superior, to medical interventions in preventing or delaying the onset of NCDs in highrisk populations ${ }^{(3)}$. In order to successfully design, implement and evaluate health and nutrition policies, policy makers require reliable data relating to determinants of NCD-related health risks, such as knowledge, perceptions and factors influencing changes in dietary behaviours ${ }^{(4)}$. Nutritional knowledge is one of the main factors influencing behaviour toward a nutritionally balanced diet, healthy lifestyle, and overall wellbeing ${ }^{(5)}$. Here, we aimed to identify the predictors of intention to eat a nutritionally balanced diet using the Theory of Planned Behaviour (TPB) among SA adults.

A cross-sectional study was conducted on a sample of SA adults aged 18 years and older. Participants were recruited via advertising on social media (Twitter and Facebook) and snowball recruitment, in December 2015 and January 2016. A questionnaire was developed and piloted in a community group ( $n=40$, not included in the main study) to ensure that the content, flow and number of questions were appropriate. The questionnaire items used were based on the TPB assessing attitudes towards behaviour (ATT), subjective norms (SN), perceived behavioural control (PBC) and actual barriers to behaviour (including knowledge) which may influence the intention of population towards nutritionally balanced diet, using five-points Likert scales ${ }^{(6)}$.

A total of 1045 participants were recruited ( $44 \%$ females). The median age of subjects was 33 years (IQR 27-40) with most $(\mathrm{n}=$ $916,88 \%)$ aged 45 or less. Half $(\mathrm{n}=540,52 \%)$ were classified as middle class. A third $(\mathrm{n}=332)$ had a background in food, nutrition or health, with $6 \%$ current students. Only $19 \%(\mathrm{n}=203)$ were aware of any nutrition \& health guidelines, with $7 \%(\mathrm{n}=76)$ reporting to follow any specific recommendations. Surprisingly, however, most $(86 \%, \mathrm{n}=779 / 902)$ intended to adopt a more nutritionallybalanced diet, with no significant difference in education levels $\left(\chi^{2}=0.648, p=0.421\right)$. There was no difference between age groups, with younger subjects ( $<45$ years) as likely to intend to make changes to their diet compared to older subjects ( $\mathrm{n}=688 / 913,75 \%$ vs. $\mathrm{n}=91 / 132,69 \%$; OR $0 \cdot 72, \mathrm{p}=0 \cdot 13)$. Among those who intended to change their behaviour, most $(\mathrm{n}=564 / 704,80 \%)$ scored high on the SN scale (perceived social pressure to engage in a behaviour towards a more balanced diet). Also, $61 \%(\mathrm{n}=375 / 619)$ scored high on the PBC scale (perceived ability to perform a given behaviour towards nutritionally balanced diet). However, only $45 \%(\mathrm{n}=347 / 779)$ answered at least 15 knowledge questions correctly out of 25 (score $60 \%$ or above). Those more knowledgeable were twice as likely to intend to change behaviour (OR 2.2, CI $95 \% 1 \cdot 4-3 \cdot 3, \mathrm{p}<0 \cdot 001)$. The awareness regarding nutrition and health guidelines was the strongest predictor of ATT, SN and ABB whilst SN towards balanced diet was the strongest predictor of subjects' intention to adopt a balanced diet.

Very few SA adults are sensitised to health and nutrition advice through the national nutrition and health guidelines (via ministry of health messaging through GPs, TV, Magazines, Adverts, or Pamphlets etc.). People with higher awareness about health and nutrition are more likely to intend to change their behaviour. Therefore, a focus on nutrition and health education interventions may benefit the SA population, along the exploration of strategies adopting a component challenging social norms.

1. Khatib O (2004) Eastern Mediterrnian Health J 10, 778-788.

2. WHO/FAO (2003) World Health Organization/Food and Agriculture Organization of the United Nations 916.

3. Elmer PJ et al. (2006) Ann of inter med 144, 485-495.

4. Paquette M-C (2005) Canadian Jour of Pub Heal 96, S15-S19.

5. Kolodinsky J et al. (2007) J of the American Dietitic Association 107, 1409-1413.

6. Ajzen I (1991) Organ Behav Hum 50, 179-211. 